\title{
Fruit Crops: Litchi
}

\author{
M. Mani
}

Mealybugs are injurious to litchi (Litchi chinensis) in Thailand, China, Japan, Indonesia, Singapore etc (Table 51.1).

Table 51.1 List of mealybugs recorded on Litchi in different countries

\begin{tabular}{l|l|l}
\hline Mealybug species & Country & Reference \\
\hline $\begin{array}{l}\text { Dysmicoccus } \\
\text { lepelleyi } \\
\text { (Betrem) }\end{array}$ & Thailand & Williams (2004) \\
\hline $\begin{array}{l}\text { Paracoccus } \\
\text { interceptus } \text { Lit. }\end{array}$ & Thailand & Williams (2004) \\
\hline $\begin{array}{l}\text { Planococcus } \\
\text { litchi Cox }\end{array}$ & $\begin{array}{l}\text { China, } \\
\text { Japan \& } \\
\text { Thailand }\end{array}$ & Ben-Dov (1994) \\
\hline $\begin{array}{l}\text { Planococcus } \\
\text { lilacinus } \\
\text { (Cockrell) }\end{array}$ & Vietnam & Williams (2004) \\
\hline $\begin{array}{l}\text { Pseudococcus } \\
\text { viburni } \\
\text { (Signoret) }\end{array}$ & - & Ben-Dov (1994) \\
\hline $\begin{array}{l}\text { Pseudococcus } \\
\text { baleiteus Lit. }\end{array}$ & $\begin{array}{l}\text { Indonesia \& } \\
\text { Thailand }\end{array}$ & Williams (2004) \\
\hline $\begin{array}{l}\text { Pseudococcus } \\
\text { cryptus Hempel }\end{array}$ & Singapore & Williams (2004) \\
\hline $\begin{array}{l}\text { Planococcus } \\
\text { litchi } \text { sp. nr. }\end{array}$ & - & Cox (1989) \\
\hline
\end{tabular}

(continued)
Table 51.1 (continued)

\begin{tabular}{l|l|l}
\hline Mealybug species & Country & Reference \\
\hline $\begin{array}{l}\text { Pseudococcus } \\
\text { comstocki } \\
\text { (Kuwana) }\end{array}$ & - & CIE (1975) \\
\hline $\begin{array}{l}\text { Pseudococcus } \\
\text { jackbeardsleyi } \\
\text { Gimpel and } \\
\text { Miller }\end{array}$ & China & $\begin{array}{l}\text { http://www. } \\
\text { plantwise.org/ } \\
\text { KnowledgeBank/ } \\
\text { Datasheet. } \\
\text { aspx?dsID=45087 }\end{array}$ \\
\hline
\end{tabular}

\section{References}

Ben-Dov Y (1994) A systematic catalogue of the mealybugs of the world (Insecta: Homoptera: Coccoidea: Pseudococcidae and Putoidae) with data on geographical distribution, host plants, biology and economic importance. Intercept Limited, Andover, $686 \mathrm{p}$

Cox JM (1989) The mealybug genus Planococcus (Homoptera: Pseudococcidae). Bull Br Museum (Nat Hist) Entomol 58(1):1-78

CIE (1975) Distribution maps of plant pests. Pseudococcus comstocki [Distribution map]. Map 338. CIE, London

Williams DJ (2004) Mealybugs of southern Asia. The Natural History Museum/Southdene SDN. BHD, London/Kuala Lumpur, 896 p

M. Mani (凶)

Indian Institute of Horticultural Research,

Bangalore 560089, India

e-mail: mmani1949@yahoo.co.in 